\title{
Mutual Coupling Reduction between Very Closely Spaced Microstrip Antennas Using CPW Structure
}

\author{
Xiao-zun $\mathrm{Ma}^{1,2}$, Ming-yang Zhao ${ }^{2}$, De-feng Zhang ${ }^{2}$ and Peng-jun $\mathrm{Liu}^{2}$ \\ ${ }^{1}$ Dept. of Electronic Engineering,Beijing University of Aeronautics and Astronautics, Beijing, China \\ ${ }^{2}$ Luoyang Electronic Equipment Test Center of China, Luoyang, Henan, China
}

\begin{abstract}
An efficient H-plane mutual coupling reduction method is introduced for very closely placed dual-element microstrip antennas operating at $2.4 \mathrm{GHz}$. A simple coplanar waveguide (CPW) structure is introduced in the ground, energy from one element is guided to another element, artificially generating new kind of mutual coupling current. By optimizing size of CPW structure to achieve purpose that new mutual coupling current cancel out with original existing mutual coupling. More than $36 \mathrm{~dB}$ isolation $\left(\left|\mathrm{S}_{21}\right|\right)$ is achieved with dual-element broadside array that distance is less than $0.024 \lambda_{0}\left(\lambda_{0}\right.$ is free space wavelength).
\end{abstract}

\section{Introduction}

Reduction mutual coupling has currently attracted considerable attention, antenna performance can be improved if mutual coupling is suppressed, such as inner-antenna spacing reduction and channel-capacity increment in MIMO application, side-lobe level (SLL) control and direction of arrival (DOA) estimation .

Coupling between antenna elements is mainly caused by surface waves and near field [1]-[3]. Near field coupling arises when one antenna is placed in near field zone of another antenna. Near field coupling becomes dominant over surface wave coupling when space between antenna elements decreases.

To degrade mutual coupling, various types of electromagnetic band-gap (EBG) have been proposed [4][7]. Because size of the cell structure of EBG is not so small, these methods are mainly applicable when substrate is thick, dielectric constant is relatively high and distance between antenna elements is not so close. Besides, EBG often involves via or two dielectric layers which increase the complexity and cost. Recently, simple and efficient methods are proposed in [8] and [9], but both of them are E-plane mutual coupling reduction.

In this letter, a novel method that etches a simple $\mathrm{CPW}$ in the ground is proposed for more degradation of H-plane mutual coupling and distance between array elements. The CPW can introduce a new coupling from the one element to another element, new mutual coupling current cancel out with original existing mutual coupling. The dual-element broadside array that distance is only $0.024 \lambda_{0}$ and the isolation is more than $36 \mathrm{~dB}$.

\section{Procedure for CPW}

\subsection{Antenna structure}

The geometry of array along with proposed CPW etched in the ground is illustrated in Fig. 1. The front of proposed antenna is in Fig. 1(a) and the back is in Fig. 1(b). This array comprises two microstrip antennas operating at $2.4 \mathrm{GHz}$. The dielectric board is $\mathrm{F}_{4} \mathrm{Bm}-2$ with relative permittivity of 2.2 , loss tangent of 0.001 and thickness of $2 \mathrm{~mm}$.

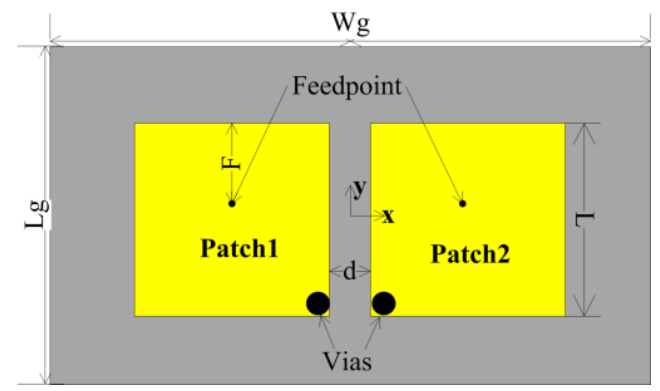

(a)

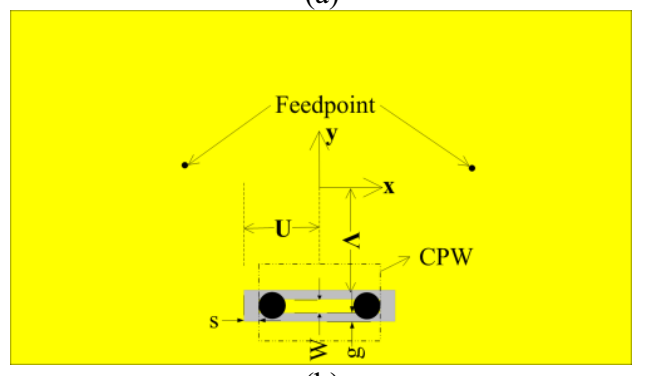

(b)

Figure 1. Geometry of the proposed patch antenna array: (a) front; (b) back. 
In Fig. 1(a), there are two square patches which are on common ground with inter-patch spacing (edge to edge) of $d$ that is about $0.024 \lambda_{0}$. The side length of the patches is $\mathrm{L}$ that is about $0.5 \lambda_{g} . F$ denotes distance between feedpoint and edge of patch. The ground size is depicted by $W g \times L g$. In Fig. 1(b), there is a CPW on the ground which is indicated by dotted bordered rectangle. In order to avoid the center line of the CPW connecting to the ground, a slot is opened at each end of the CPW. Through the via, CPW is connected to patches. $U$ and $V$ denote respectively the distance between the CPW and the $y$-axis or $x$-axis. $W$ and $g$ denote the width of center line and gaps of the CPW respectively. The width of the slot is s.

\subsection{Parametric studies}

CST Microwave Studio was used to study parameters of the proposed structure. Through parameters sweeps and optimization, $U$ and $V$ were found important in this design. The results of parametric studies are depicted in Fig. 2 and Fig. 3.

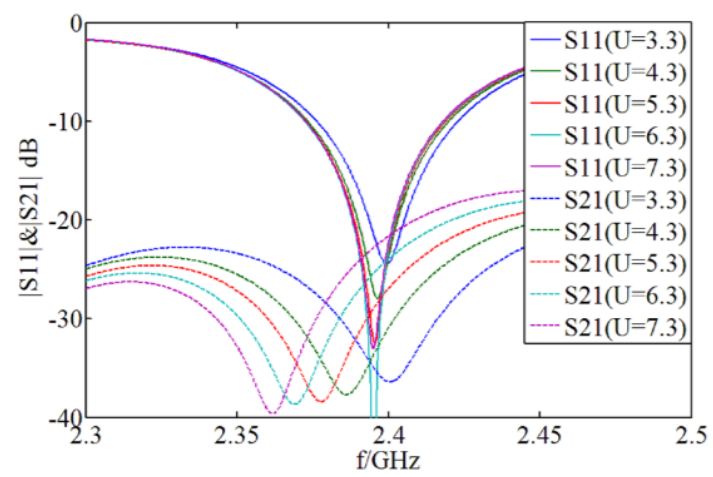

Figure 2. S-parameters varying with $\mathrm{U}$ (unit of $\mathrm{U}$ : $\mathrm{mm}$ ).

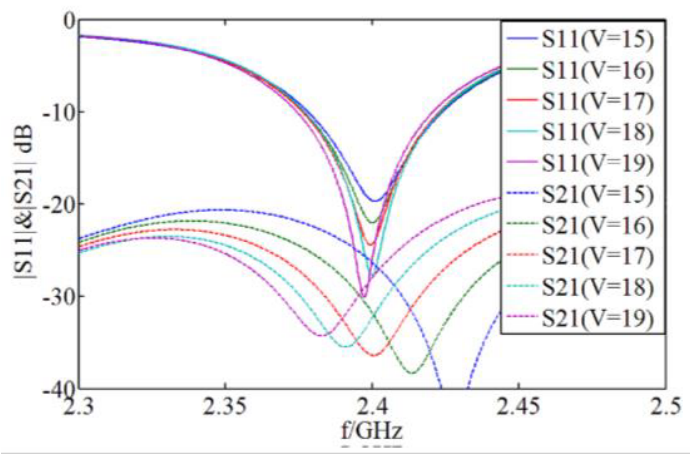

Figure 3. S-parameters varying with V(unit of V: $\mathrm{mm}$ ).

Fig. 2 shows the S-parameters along with the parameter $U$. when the other parameters are set to $d=3$ $\mathrm{mm}, V=17 \mathrm{~mm}, F=12 \mathrm{~mm}, W=0.8 \mathrm{~mm}, g=0.4 \mathrm{~mm}$, $L g=73.5 \mathrm{~mm}, W g=116 \mathrm{~mm}, s=0.8 \mathrm{~mm}, \mathrm{~L}=40 \mathrm{~mm}$. The solid line shows the $\mathrm{S}_{11}$, while the dotted line shows the $\mathrm{S}_{21}$ (the same below). As shown, the operating frequency changes very small along with $U$, while the frequency of the minimum value of $S_{21}$ moves to lower frequency when $U$ increased. This is because that the field distribution is different at different place of patches, which affects the new coupling field that CPW introduces.
Fig. 3 shows the S-parameters along with the parameter $V$. The parameter $V$ has almost the same effect of $U$, but the minimum value of $\mathrm{S}_{21}$ is increased when $V$ is increased.

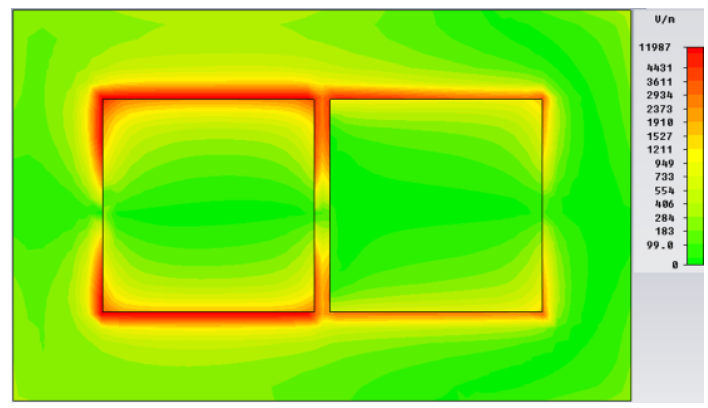

(a)

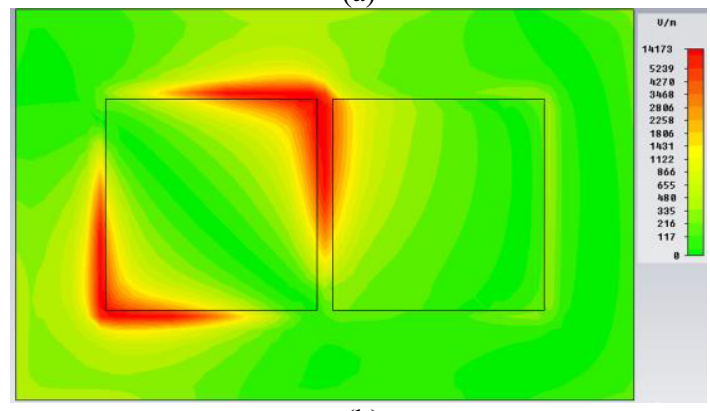

(b)

Figure 4. Absolute electric-field distribution:(a) the normal; (b)the proposed.

\section{Result and discussion}

In order to understand the mechanism of the proposed method, the electric-field distribution comparisons between the proposed antennas and normal microstrip antennas are shown in Fig. 4(Patch 1 is excited while the Patch 2 is terminated with a $50-\Omega$ impedance). The mutual coupling for normal antenna elements is serious with respect to the proposed antennas. In Fig. 4 (b), the electric-field distribution along the $x$-axis direction is uneven. At the position and the symmetric position of vias, there is no electric-field distribution. In the proposed structure, the vias worked as a probe, which is connected to the CPW. The energy from Patch 1 is guided to Patch 2 through the vias and the CPW, which offsets the original existing coupling. To Patch 2 , it is that the conduction current (introduced by CPW) and the displacement current (mainly caused by original existing near field coupling) offset each other.

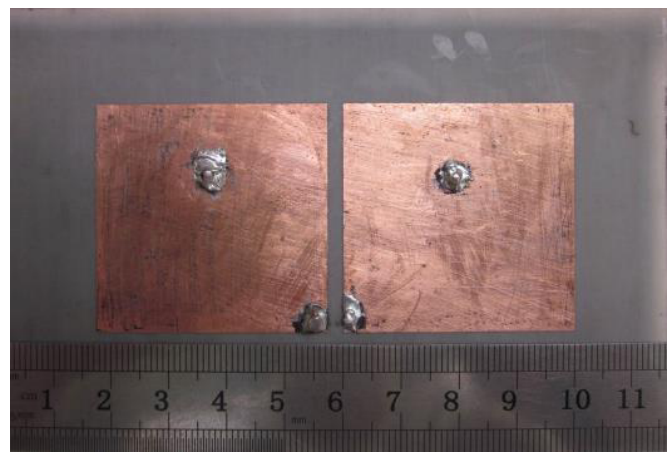

(a) 


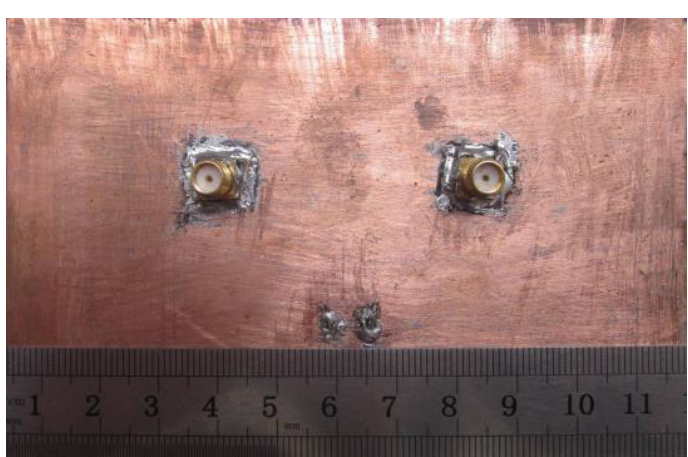

(b)

Figure 5. Photograph of the two fabricated prototypes:(a) front; (b) back.

Finally, proposed designs were fabricated and measured using Agilent vector network analyzer-8510B. Photographs of the fabricated prototypes are shown in Fig. 5. Taking into account processing convenience, throughvias are replaced by soldered conductor pillars. In fact, the CPW is so small that is difficult to be found in the photograph.

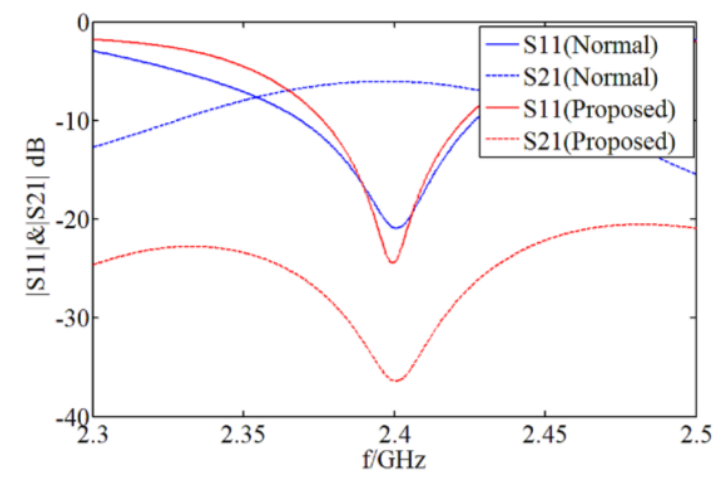

Figure 6. S-parameters comparison of normal and proposed microstrips.

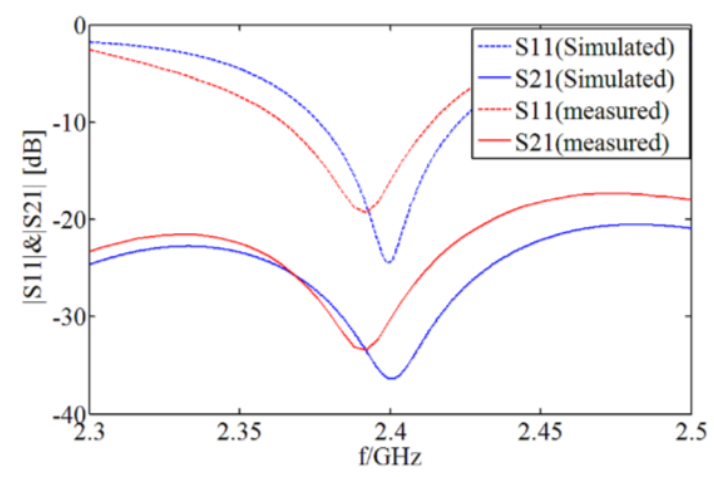

Figure 7. Simulated and measured S-parameters.

Results of S-parameters of the proposed structure in comparison to the normal structure are presented in Fig. 6. As shown, the proposed structure provides a significant improvement of isolation about $30 \mathrm{~dB}$ compared to conventional normal microstrip antennas. It should be noted that patch dimensions and feed-point position have been done a slight adjustment. Fig. 7 shows comparisons between simulation and measured results. Compared to the simulation, the measurement results are slightly worse and there is a little frequency offset, which are mainly caused by the machining error.
Radiation patterns in H-plane and E-plane of proposed structure in comparison to single patch antenna and normal patch antennas at $2.4 \mathrm{GHz}$ are presented in Fig. 8. For the H-plane, the main lobe direction of array element of proposed antennas is slightly different from the single, while main lobe direction of array element of normal antennas is significantly different. The former may be due to the asymmetry of the ground plane ; the later is because that radiation of coupling patch offsets or enhances original radiation. For E-plane, these simulation results don't show any significant differences, but directivity of normal antennas is smaller than others.

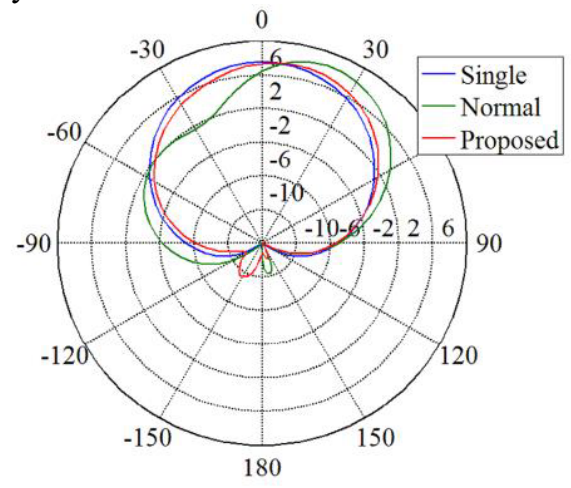

(a)

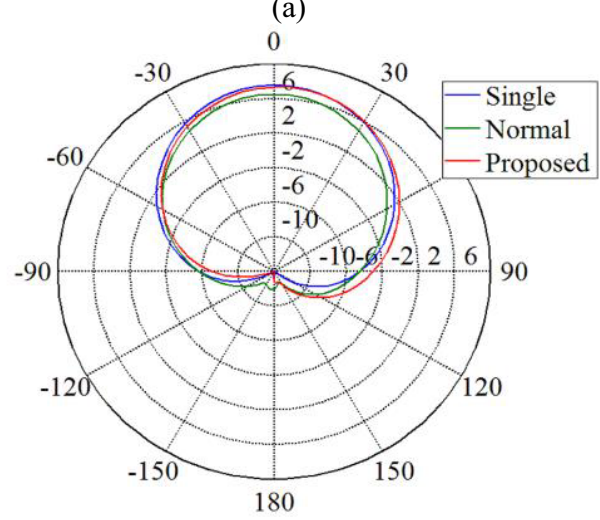

(b)

Figure 8. Radiation patterns of single, normal and proposed antennas at 2.4GHz:(a) H-plane (xz-plane). (b)E-plane (yzplane).

Finally, to further validate the radiation effects of the proposed structure, simulated values of the directivity, main lobe direction and side lobe lever (SLL) of the designs compared to the single patch antenna and normal antennas are summarized in Table 1 . The table shows that the proposed structure, compared to normal structure, improves significantly the side lobe lever and main lobe direction. At the same time, the directivity of the proposed structure is close to the single patch.

Table 1. Comparison of radiation.

\begin{tabular}{|c|c|c|c|c|c|c|}
\hline \multirow{2}{*}{ Design } & \multicolumn{2}{|c|}{ Directivity(dBi) } & \multicolumn{2}{|c|}{ Main lobe direction $\left({ }^{\circ}\right)$} & \multicolumn{2}{|c|}{ SLL(dB) } \\
\cline { 2 - 7 } & $\mathrm{xz}$ & $\mathrm{yz}$ & $\mathrm{xz}$ & $\mathrm{yz}$ & $\mathrm{xz}$ & $\mathrm{yz}$ \\
\hline Single & 7.5 & 7.5 & 0 & 0 & -20.6 & -20.6 \\
\hline Normal & 8.4 & 6.5 & 21 & 0 & -6.4 & -18.3 \\
\hline Propo & 7.5 & 7.3 & 10 & 3 & -16.9 & -20.0 \\
\hline
\end{tabular}




\section{Summary}

In this letter, an efficient but simple technique is proposed for more reduction of the mutual coupling and distance between the antenna array elements. Through a simple CPW on the ground plane, more than $36 \mathrm{~dB}$ isolation is achieved with inter-antenna spacing of less than $0.024 \lambda_{0}$. The technique is suitable for MIMO antennas and compact arrays.

\section{References}

1. A. Bhattacharyya, Characteristics of space and surface waves in a multilayered structure, IEEE Trans. Antennas Propag., 38, 8, pp.1231-1238, (1990).

2. D. Jackson, J. Williams, A. Bhattacharyya, R. Smith, S. Buchheit, and S. Long, Microstrip patch designs that do not excite surface waves, IEEE Trans. Antennas Propag., 41, 8, pp. 1026-1037, (1993).

3. M. M. Bait-Suwailam, O. F. Siddiqui and O. M. Ramahi, Mutual Coupling Reduction Between Microstrip Patch Antennas Using SlottedComplementary Split-Ring Resonators, IEEE Antennas Wireless Propag. Lett., 9, pp.876-878, (2010).

4. M. Coulombe, S. F. Koodiani and C. Caloz, Compact Elongated Mushroom (EM)-EBG Structure for Enhancement of Patch Antenna Array, IEEE
Trans. Antennas Propag., 58, 4, pp. 1076-1086, (2010).

5. F. Yang and Y. Rahmat-Samii, Microstrip Antennas Integrated With Electromagnetic BandGap (EBG) Structures: A Low Mutual Coupling Design for Array Applications, IEEE Trans. Antennas Propag., 51, 10, pp. 2936-2946, (2003).

6. E. Rajo-Iglesias, Ó. Quevedo-Teruel and L. InclánSánchez, Mutual Coupling Reduction in Patch Antenna Arrays by Using a Planar EBG Structure and a Multilayer Dielectric Substrate, IEEE Trans. Antennas Propag., 56, 6, pp. 1648-1655, (2008).

7. H. S. Farahani, M. Veysi, M. Kamyab and A. Tadjalli, Mutual Coupling Reduction in Patch Antenna Arrays Using a UC-EBG Superstrate, IEEE Antennas Wireless Propag. Lett., 9, pp.57-58, (2010).

8. A. Habashi, J. Nourinia and C. Ghobadi, et al. Mutual Coupling Reduction Between Very Closely Spaced Patch Antennas Using Low-Profile Folded Split-Ring Resonators (FSRRs), IEEE Antennas Wireless Propag. Lett., 10, pp.862-865, (2011).

9. J. O. Yang, F. Yang and Z.M. Wang, et al. Reducing Mutual Coupling of Closely Spaced Microstrip MIMO Antennas for WLAN Application, IEEE Antennas Wireless Propag. Lett., 10, pp.310-313, (2011). 